Bull. Austral. Math. Soc.

$65 \mathrm{~L} 10,65 \mathrm{~L} 50$

VOL. 41 (1990) [75-86]

\title{
CONTINUOUS AND NUMERICAL ANALYSIS OF A BOUNDARY SHOCK PROBLEM
}

\author{
Relja Vulanović
}

\begin{abstract}
A quasilinear singularly perturbed boundary value problem whose solution has a shock layer is investigated. Estimates of the derivatives of the solution are derived. Based on these estimates, a new independent variable is introduced. Then the transformed problem is solved numerically using finite - difference schemes. The transformation corresponds to solving the original problem on a mesh which is dense in the layer. The linear convergence uniform in the perturbation parameter is proved in the discrete $L^{1}$ norm. Numerical results show uniform pointwise convergence too.
\end{abstract}

\section{INTRODUCTION}

The aim of the paper is to construct a uniform numerical method for solving the following quasilinear boundary value problem with a small positive perturbation parameter $\varepsilon$ :

$$
-\varepsilon u^{\prime \prime}-u b(u) u^{\prime}+u c(x, u)=0, u(0)=0, u(1)=B>0
$$

Under appropriate conditions, which we shall state in the next Section, it follows that there exists a unique solution, $u_{\varepsilon}$, to the problem (1). We are interested in the boundary shock layer behaviour of $u_{e}$, in the sense of [2]. In Section 2 we derive estimates of the derivatives of $u_{\varepsilon}$. They are necessary to prove that our numerical method is uniform in $\varepsilon$.

The numerical method is given in Section 3. Essentially, it uses finite-difference (upwind) schemes on a special non-equidistant mesh which is dense in the layer. However, the mesh is introduced indirectly: the problem (1) is transformed by changing the independent variable and then the resulting problem is solved numerically on an equidistant mesh. This approach can be found in [3], as well as in some earlier papers of these authors, and in [10]. We use here the same transformation as in [10], where the non-turning point case of a quasilinear singularly perturbed boundary value problem was considered. Our main result is the uniform (that is, uniform in $\varepsilon$ ) first order

\section{Received 7 February 1989}

This work was supported by the NSF and ZAMTES of Vojvodina through funds made available to the U.S.-Yugoslav Joint Board on Scientific and Technological Cooperation, Project No. JF 799.

Copyright Clearance Centre, Inc. Serial-fee code: 0004-9729/90 \$A2.00+0.00. 
convergence of the numerical solution towards the restriction of $u_{e}$ on the mesh, in the discrete $L^{1}$ norm. The result follows from the principle: uniform stability + uniform consistency $\Rightarrow$ uniform convergence. Uniform stability can be proved in the discrete $L^{1}$ norm (see $[1,4,5,6]$ ) and this is the reason why this norm is used. But, on the other hand, we are not able to prove uniform pointwise convergence, which is present, as numerical examples in Section 4 show.

This paper might be regarded as a step towards uniform numerical methods for more general problems whose solutions have interior shock layers, such as the Lagerstrom-Cole model problem, see [2]. In these cases the problem of locating shocks has to be resolved.

Singularly perturbed quasilinear problems have been solved numerically in $[1,7]$ and $[4,5,6]$ (the case $\varepsilon=0$ ), just to mention some of the papers. Note that none of these papers deals with the proof of uniform convergence in the above sense.

\section{Estimates of the Derivatives}

Thus we shall consider the problem:

$$
\begin{gathered}
T u=-\varepsilon u^{\prime \prime}-u b(u) u^{\prime}+u c(x, u)=0, x \in I=[0,1], \\
R u=(u(0), u(1))=(0, B), B>0,
\end{gathered}
$$

where $\quad=d / d x, 0<\varepsilon \leqslant \varepsilon^{*}<1$ (usually $\varepsilon^{*}<<1$ ).

Let

$$
U=[0, B] \text {. }
$$

We assume

$$
\begin{gathered}
b \in C^{2}(U), \quad c \in C^{2}(I \times U) \\
c^{*} \geqslant c(x, u) \geqslant c_{*} \geqslant 0, \quad x \in I, \quad u \in U, \\
b^{*} \geqslant b(u) \geqslant b_{*}>0, \quad u \in U .
\end{gathered}
$$

Because of (4) 0 and $B$ are lower and upper solutions to (2), thus the problem (2) has a solution, $u_{\varepsilon} \in C^{4}(I)$, and

$$
u_{c}(x) \in U, x \in I \text {. }
$$

Furthermore, from (4) we have $u_{\varepsilon}^{\prime}(x) \geqslant 0$ for $x \in I$.

The following inequality will be of interest:

$$
B>Q=\left\{b^{*} c^{*}+\left[b^{*} c^{*}\left(b^{*} c^{*}-b_{*} c_{*}\right)\right]^{1 / 2}\right\} /\left(b^{*} b_{*}\right) .
$$

We suppose that the conditions (3-6) hold throughout the section.

In this and the next Section we shall denote by $M$ any (in the sense of $O(1)$ ) positive constant which is independent of $\varepsilon$. In particular, some of these constants will be denoted by $m, m^{\prime}, m_{0}$ et cetera. 
LEMMA 1. $u_{\varepsilon}^{\prime}(0) \geqslant m_{1} / \varepsilon$.

Proof: First we show that

$$
u_{e}(x) \leqslant u^{*}(x)=\left(c_{*} / b^{*}\right)(x-1)+B, x \in I .
$$

Let $z_{e}$ be the solution to the following special case of the problem (2):

$$
\tilde{T} u=-\varepsilon u^{\prime \prime}-b^{*} u u^{\prime}+c_{*} u=0, \quad R u=(0, B) .
$$

Because of (4) and (5) we have

$$
\bar{T} u_{\varepsilon}=\left(b\left(u_{\varepsilon}\right)-b^{*}\right) u_{\varepsilon}^{\prime}+\left(c_{*}-c\left(x, u_{\varepsilon}\right)\right) u_{\varepsilon} \leqslant 0, \cdot x \in I,
$$

and since the operator $(\tilde{T}, R)$ is inverse monotone, we get

$$
u_{\varepsilon}(x) \leqslant z_{\varepsilon}(x), \quad x \in I .
$$

Now introduce the linear operator:

$$
L u=-\varepsilon u^{\prime \prime}-b^{*} z_{\varepsilon} u^{\prime}
$$

We have

$$
L\left(u^{*}-z_{\varepsilon}\right)=0, \quad\left(u^{*}-z_{\varepsilon}\right)(1)=0,
$$

and (6) implies

$$
u^{*}(0)>0 \text {. }
$$

Thus by the inverse monotonicity of $(L, R)$ we get

$$
z_{\varepsilon}(x) \leqslant u^{*}(x), \quad x \in I,
$$

which together with (8) completes the proof of (7).

Now rewrite (2a) in the form:

$$
\begin{gathered}
-\varepsilon u^{\prime \prime}-f(u)^{\prime}+u c(x, u)=0, \\
f(u)=\int_{0}^{u} s b(s) d s
\end{gathered}
$$

and integrate $(9 \mathrm{a})$ from 0 to 1 . Using $u_{e}^{\prime}(1) \geqslant 0$ we get

$$
\begin{gathered}
\varepsilon u_{e}^{\prime}(0) \geqslant f(B)-\int_{0}^{1} u_{e}(x) c\left(x, u_{e}(x)\right) d x \geqslant \\
f(B)-c^{*} \int_{0}^{1} u^{*}(x) d x=\left(b_{*} / 2\right) B^{2}-c^{*} B+c^{*} c_{*} /\left(2 b^{*}\right)=m_{1} .
\end{gathered}
$$

We have $m_{1}>0$ because of $(6)$. 
Remark. Condition (6) implies $B>c^{*} / b_{*}$ which by Peano's theorem guarantees the existence of a solution $u_{R}$ to the reduced problem

$$
-u b(u) u^{\prime}+u c(x, u)=0, x \in I, u(1)=B
$$

Moreover, $u_{R}^{\prime}(x) \geqslant 0$ for $x \in I$, and $u_{R}(0)>0$, hence $u_{\varepsilon}$ has a layer at $x=0$.

The condition (6) is artificial to some extent. Note, however, that in the constant coefficient case $\left(b=b^{*}=b_{*}, c=c^{*}=c_{*}\right)$ it reduces to

$$
B>c / b
$$

which is the necessary condition for existence of a boundary layer at $x=0$. If $B \leqslant c / b$, the interior crossing phenomenon occurs, see [2].

Let

$$
F(x)=\int_{0}^{x} u_{e}(s) b\left(u_{e}(s)\right) d s
$$

We have:

LEMMA 2. $u_{\varepsilon}^{\prime}(x) \leqslant M\left(1+\varepsilon^{-1} \exp (-F(x) / \varepsilon)\right), x \in I$.

Proof: From (2a) it follows that

$$
\varepsilon\left(\exp (F(x) / \varepsilon) u_{e}^{\prime}(x)\right)^{\prime}=u_{\varepsilon}(x) c\left(x, u_{\varepsilon}(x)\right) \exp (F(x) / \varepsilon) .
$$

Expressing $u_{e}^{\prime}(x)$ by integration we get

$$
\begin{aligned}
u_{\varepsilon}^{\prime}(x) & =\left[\varepsilon^{-1} \int_{0}^{x} g(t) u_{\varepsilon}(t) b\left(u_{e}(t)\right) \exp (F(t) / \varepsilon) d t+u_{e}^{\prime}(0)\right] \exp (-F(x) / \varepsilon) \\
& =\left[g(\theta)(\exp (F(x) / \varepsilon)-1)+u_{e}^{\prime}(0)\right] \exp (-F(x) / \varepsilon),
\end{aligned}
$$

where $g(x)=c\left(x, u_{\varepsilon}(x)\right) / b\left(u_{\varepsilon}(x)\right), \theta \in(0, x)$. Thus:

$$
u_{\varepsilon}^{\prime}(x)=g(\theta)(1-\exp (-F(x) / \varepsilon))+u_{e}^{\prime}(0) \exp (-F(x) / \varepsilon) .
$$

Now integrate (10) from 0 to 1 and express $u_{\varepsilon}^{\prime}(0)$. It follows:

$$
u_{e}^{\prime}(0) \leqslant M / J, \quad J=\int_{0}^{1} \exp (-F(x) / \varepsilon) d x .
$$

Since

$$
J \geqslant \int_{0}^{1} \exp \left(-b^{*} B x / \varepsilon\right) d x=\varepsilon\left(1-\exp \left(-b^{*} B / \varepsilon\right)\right) /\left(b^{*} B\right),
$$

we get

$$
u_{\varepsilon}^{\prime}(0) \leqslant M / \varepsilon
$$

which together with (10) gives the assertion. 
LEMMA 3 .

$$
F(x) \geqslant m_{2} x^{2} / \varepsilon \text { for } x \in[0, \varepsilon] \text {, }
$$

$$
F(x) \geqslant m_{3}(x-\varepsilon) \text { for } x \in[\varepsilon, 1] \text {. }
$$

Proof: Let $x \in[0, \varepsilon]$. Then we use

$$
u_{\varepsilon}(x)=x u_{\varepsilon}^{\prime}(\sigma), \quad \sigma \in(0, \varepsilon)
$$

From (10) it follows that

$$
\begin{aligned}
u_{e}^{\prime}(\sigma) & \geqslant u_{e}^{\prime}(0) \exp (-F(\sigma) / \varepsilon) \\
& \geqslant m_{1} \exp \left(-b^{*} B\right) / \varepsilon=m_{4} / \varepsilon
\end{aligned}
$$

where we have used Lemma 1. Thus from (12) we get

$$
u_{\varepsilon}(x) \geqslant m_{4} x / \varepsilon, \quad x \in[0, \varepsilon],
$$

and (11a) follows with $m_{2}=b_{*} m_{3} / 2$.

Now let $x \in[\varepsilon, 1]$. We have

$$
u_{\varepsilon}(x) \geqslant u_{e}(\varepsilon)=\varepsilon u_{\varepsilon}^{\prime}(\gamma), \quad \gamma \in(0, \varepsilon)
$$

thus, using the above technique we get

$$
u_{e}(x) \geqslant m_{4}
$$

Now

$$
F(x) \geqslant \int_{e}^{x} u_{e}(s) b\left(u_{e}(s)\right) d s \geqslant b_{*} m_{4}(x-\varepsilon),
$$

and (11b) is proved with $m_{3}=b_{*} m_{4}$.

LEMMA 4. $\left|u_{\varepsilon}^{\prime \prime}(x)\right| \leqslant M\left(1+\varepsilon^{-3} x \exp (-F(x) / \varepsilon)\right), x \in I$.

Proof: Differentiate (2a) and use the same technique as in the proof of Lemma 2 to obtain:

$$
u_{e}^{\prime \prime}(x)=\varepsilon^{-1} \exp (-F(x) / \varepsilon) \int_{0}^{x} G(t) \exp (F(t) / \varepsilon) d t
$$

(note that $u_{e}^{\prime \prime}(0)=0$ ) where from Lemma 2 it follows that

$$
|G(t)| \leqslant M\left(1+\varepsilon^{-1} \exp (-F(t) / \varepsilon)+\varepsilon^{-2} \exp (-2 F(t) / \varepsilon)\right) .
$$


Thus:

$$
\left|u_{\varepsilon}^{\prime \prime}(x)\right| \leqslant M \varepsilon^{-1} \exp (-F(x) / \varepsilon) \int_{0}^{x}\left[\varepsilon^{-2}+\exp (F(t) / \varepsilon)\right] d t
$$

and in order to prove the lemma we have to show that

$$
S=\varepsilon^{-1} \int_{0}^{x} \exp ((F(t)-F(x)) / \varepsilon) d t \leqslant M
$$

Let $x \in[0, \varepsilon]$. Then obviously $S \leqslant 1$. Now let $x \in[\varepsilon, 1]$. We have

$$
S \leqslant 1+\int_{\varepsilon}^{x} \exp ((F(t)-F(x)) / \varepsilon) d t
$$

Since for $\varepsilon \leqslant t \leqslant x \leqslant 1$ we have

$$
F(t)-F(x) \leqslant b_{*} m_{4}(t-x)
$$

where $m_{4}$ is the same as in (13), from (15) we get (14).

Theorem 1 .

$$
\begin{gathered}
\left|u_{\varepsilon}^{(k)}(x)\right| \leqslant M\left(1+\varepsilon^{-k} \exp (-m x / \varepsilon)\right), \\
x \in I, \quad k=1,2,3 .
\end{gathered}
$$

Proof: The proof of the cases $k=1,2$ follows immediately from Lemmas 2, 3 and 4, with apppropriate $M$ and $m$. It remains to prove the case $k=3$. First differentiate (2a) and use Lemma 2 to get $\left|u_{e}^{(3)}(0)\right| \leqslant M \varepsilon^{-3}$. Then diffenentiate (2a) once more and obtain:

$$
\begin{aligned}
\left|u_{\varepsilon}^{(3)}(x)\right| & \leqslant M \exp (-F(x) / \varepsilon)\left\{\varepsilon^{-3}+\varepsilon^{-1} \int_{0}^{x}\left[1+\varepsilon^{-1} \exp (-F(t) / \varepsilon)\right.\right. \\
& +\varepsilon^{-2} \exp (-2 F(t) / \varepsilon)+\varepsilon^{-3} \exp (-3 F(t) / \varepsilon)+\varepsilon^{-3} t \exp (-F(t) / \varepsilon) \\
& \left.\left.+\varepsilon^{-4} t \exp (-2 F(t) / \varepsilon)\right] \exp (F(t) / \varepsilon) d t\right\} \\
\leqslant & M \exp (-F(x) / \varepsilon)\left\{\varepsilon^{-3}+\varepsilon^{-1} \int_{0}^{x}\left[\varepsilon^{-3}+\varepsilon^{-4} t+\exp (F(t) / \varepsilon)\right] d t\right\} \\
\leqslant & M\left(\varepsilon^{-3}+\varepsilon^{-4} x+\varepsilon^{-5} x^{2}\right) \exp (-F(x) / \varepsilon)+S,
\end{aligned}
$$

where $S$ is the same as in (14), and the assertion follows because of Lemma 3. 


\section{The Numerical method}

We shall give here the numerical method for the problem (2). Additionally, we have to assume

$$
(u c(x, u))_{u} \geqslant c_{1}>0, x \in I, u \in U
$$

Let

$$
\lambda(t)=\left\{\begin{array}{r}
\omega(t)=\beta \varepsilon t /(\gamma-t), t \in[0, \alpha] \\
\pi(t)=\delta(t-\alpha)^{3}+\omega^{\prime \prime}(\alpha)(t-\alpha)^{2} / 2 \\
\quad+\omega^{\prime}(\alpha)(t-\alpha)+\omega(\alpha), t \in[\alpha, 1]
\end{array}\right.
$$

where $\alpha \in(0,1)$ is given and $\gamma=\alpha+\varepsilon^{1 / 4}$. Obviously, $\lambda \in C^{2}(I)$ and $\lambda \in C^{\infty}[0, \alpha]$, $\lambda \in C^{\infty}[\alpha, 1]$. Coefficient $\delta$ is determined from the condition $\pi(1)=1$, while coefficient $\beta$ is chosen to satisfy:

$$
0<\beta \varepsilon^{1 / 4}\left(\gamma(1-\alpha)^{2}+\varepsilon^{1 / 4} \gamma(1-\alpha)+\varepsilon^{1 / 2} \alpha\right) \leqslant 1,
$$

so that $\delta \geqslant 0$ and $\pi^{\prime \prime}(t)$ is non-decreasing. From this we have

hence

and obviously

$$
\begin{aligned}
\pi^{\prime \prime}(t) \geqslant \pi^{\prime \prime}(\alpha) & =\omega^{\prime \prime}(\alpha)>0, \quad t \in[\alpha, 1], \\
\pi^{\prime}(t) \geqslant \pi^{\prime}(\alpha) & =\omega^{\prime}(\alpha)>0, \quad t \in[\alpha, 1],
\end{aligned}
$$$$
\omega^{\prime}(t)>0 \text { for } t \in[0, \alpha] \text {. }
$$

Thus, the function $\lambda$ can be used to introduce a new independent variable $t$ via $x=$ $\lambda(t)$. Let $y(t)=u(\lambda(t))$. Then the transformed problem (9), (2b) reads:

$$
\begin{gathered}
P y=-\varepsilon\left(\mu(t) y^{\prime}\right)^{\prime}-f(y)^{\prime}+q(t, y)=0, \\
R y=(0, B)
\end{gathered}
$$

where ' $=d / d t$

$$
\begin{aligned}
\mu(t) & =1 / \lambda^{\prime} /(t), \\
q(t, y) & =y c(\lambda(t), y) \lambda^{\prime}(t) .
\end{aligned}
$$

The same function $\lambda$ was used in [10]. Its part $\omega$ is a certain modification of the inverse of the boundary layer function $\exp (-m x / \varepsilon)$, see $[8,9,3]$.

Let $I_{h}$ be an equidistant mesh with points

$$
t_{i}=i h, i=0,1, \ldots, n, \quad h=1 / n, \quad n \in N \quad(n \geqslant 4),
$$

and let

$$
t_{i+1 / 2}=t_{i} \pm h / 2, \quad i=1,2, \ldots, n-1 \text {. }
$$


We form the discrete problem corresponding to (17):

$$
P_{h} w_{h}=0 \text {, }
$$

where $w_{h}=\left[w_{1}, w_{2}, \ldots, w_{n-1}\right]^{T} \in R^{n-1}$ is a mesh function on $I_{h} \backslash\{0,1\}$ and $P_{h}: \mathbf{R}^{n-1} \rightarrow \mathrm{R}^{n-1}$ is

$$
\begin{gathered}
P_{h} w_{1}=\varepsilon h^{-2}\left[\left(\mu\left(t_{1 / 2}\right)+\mu\left(t_{3 / 2}\right)\right) w_{1}-\mu\left(t_{3 / 2}\right) w_{2}\right] \\
\quad-h^{-1}\left[f\left(w_{2}\right)-f\left(w_{1}\right)\right]+q\left(t_{1}, w_{1}\right) \\
P_{h} w_{i}=\varepsilon h^{-2}\left[-\mu\left(t_{i-1 / 2}\right) w_{i-1}+\left(\mu\left(t_{i-1 / 2}\right)+\mu\left(t_{i+1 / 2}\right)\right) w_{i}\right. \\
\left.\quad-\mu\left(t_{i+1 / 2}\right) w_{i+1}\right]-h^{-1}\left[f\left(w_{i+1}\right)-f\left(w_{i}\right)\right] \\
\quad+q\left(t_{i}, w_{i}\right), i=2,3, \ldots, n-2, \\
P_{h} w_{n-1}=\varepsilon h^{-2}\left[-\mu\left(t_{n-3 / 2}\right) w_{n-2}+\left(\mu\left(t_{n-3 / 2}\right)+\mu\left(t_{n-1 / 2}\right)\right) w_{n-1}\right. \\
\left.\quad-\mu\left(t_{n-1 / 2}\right) B\right]-h^{-1}\left[f(B)-f\left(w_{n-1}\right)\right]+q\left(t_{n-1}, w_{n-1}\right) .
\end{gathered}
$$

Let $\|\cdot\|_{1}$ denote the usual vector (matrix) norm in $R^{n-1}\left(R^{n-1, n-1}\right)$. Let $e_{h}=$ $[1,1, \ldots, 1]^{T} \in R^{n-1}$ and let

$$
U_{h}=\left\{w_{h} \in \mathrm{R}^{n-1}: B e_{h} \geqslant w_{h} \geqslant 0\right\}
$$

(the inequality sign in $\mathbf{R}^{n-1}$ should be understood componentwise). We have:

THEOREM 2. Let (3-5) and (16) hold. Then in $U_{h}$ there exists a unique solution $w_{e, h}$ to the discrete problem (18). Moreover, for any $w_{h}, v_{h} \in U_{h}$ the following stability inequality holds

$$
\sum_{i=1}^{n-1} \lambda^{\prime}\left(t_{i}\right)\left|w_{i}-v_{i}\right| \leqslant c_{1}^{-1}\left\|P_{h} w_{h}-P_{h} v_{h}\right\|_{1} .
$$

Proof: Let us prove (19). For $w_{h}, v_{h} \in U_{h}$ we set $z_{i}=w_{i} \lambda^{\prime}\left(t_{i}\right), g_{i}=v_{i} \lambda^{\prime}\left(t_{i}\right)$, $i=1,2, \ldots, n-1$, and introduce a new operator:

$$
\tilde{P}_{h} z_{i}=P_{h}\left(z_{i} / \lambda^{\prime}\left(t_{i}\right)\right), \quad i=1,2, \ldots, n-1 .
$$

Then the Frechet derivative of $\tilde{P}_{h}$ satisfies:

$$
\left\|\left(\tilde{P}_{h}^{\prime}\left(z_{h}\right)\right)^{-1}\right\|_{1} \leqslant 1 / c_{1},
$$

see $[5,6]$. Indeed, $\tilde{P}_{h}^{\prime}\left(z_{h}\right)$ is an $L$-matrix and

$$
\begin{gathered}
\left(\tilde{P}_{h}^{\prime}\left(z_{h}\right)\right)^{T} e_{h} \geqslant c_{1} e_{h} . \\
\left\|z_{h}-g_{h}\right\|_{1} \leqslant c_{1}^{-1}\left\|\tilde{P}_{h} z_{h}-\tilde{P}_{h} g_{h}\right\|_{1},
\end{gathered}
$$

It follows that 
and (19) is immediate. The inequalities

$$
P_{h}\left(B e_{h}\right) \geqslant 0 \geqslant P_{h} 0,
$$

complete the proof.

Let us now introduce the norm:

where

$$
\left\|w_{h}\right\|_{h}=\sum_{i=1}^{n-1} \bar{h}_{i}\left|w_{i}\right|,
$$

$$
\begin{aligned}
& \bar{h}_{i}=\left(x_{i+1}-x_{i-1}\right) / 2, i=1,2, \ldots, n-1, \\
& x_{i}=\lambda\left(t_{i}\right), i=0,1, \ldots, n .
\end{aligned}
$$

This is the standard $L^{1}$ discrete norm, see [1]. Let $w_{\varepsilon, h}$ be as in Theorem 2 and let

$$
u_{\varepsilon, h}=\left[u_{\varepsilon}\left(x_{1}\right), u_{\varepsilon}\left(x_{2}\right), \ldots, u_{\varepsilon}\left(x_{n-1}\right)\right]^{T}
$$

Moreover, in this section we let the constants $M$ be independent of $h$ as well. Then we have:

THEOREM 4. Let (3-6) and (16) hold and let the function $\lambda$ be given with $\alpha=t_{j}$ for some $j \in(1,2, \ldots, n-1)$. Then:

$$
\left\|u_{\varepsilon, h}-w_{\varepsilon, h}\right\|_{h} \leqslant M h
$$

Proof: From (19) we have

$$
\sum_{i=1}^{n-1} \lambda^{\prime}\left(t_{i}\right)\left|u_{\varepsilon}\left(x_{i}\right)-w_{e, i}\right| \leqslant c_{1}^{-1}\left\|r_{h}\right\|_{1},
$$

where

$$
r_{h}=P_{h} u_{\varepsilon, h}
$$

The components of the consistency-error-vector $r_{h}$ are:

$$
r_{i}=P_{h} y_{\varepsilon}\left(t_{i}\right)-\left(P y_{\varepsilon}\right)\left(t_{i}\right), \quad i=1,2, \ldots, n-1 \text {, }
$$

where $y_{\varepsilon}(t)=u_{\varepsilon}(\lambda(t))$. The following estimates are proved in [10]:

$$
\begin{gathered}
\varepsilon\left|\mu^{(k)}(t)\right| \leqslant M, k=0,1,2, \\
\left|y_{e}^{(k)}(t)\right| \leqslant M, k=1,2,3,
\end{gathered}
$$


where $t \in\left(t_{i-1}, t_{i}\right), i=1,2, \ldots, n$, (note that these derivatives exist in $\left(t_{i-1}, t_{i}\right)$ ). It follows that

$$
\left|r_{i}\right| \leqslant M h, \quad i=1,2, \ldots, n-1 \text {. }
$$

Furthermore, it is easy to prove that

$$
\left|h \lambda^{\prime}\left(t_{i}\right)-\bar{h}_{i}\right| \leqslant M h^{3}, \quad i=1,2, \ldots, n-1
$$

and, obviously:

$$
\left|u_{\varepsilon}\left(x_{i}\right)-w_{\varepsilon, i}\right| \leqslant M, \quad i=1,2, \ldots, n-1 \text {. }
$$

Now multiply (21) by $h$ and use (22) to obtain (20).

\section{NUMERICAL RESULTS}

Let us consider the problem

$$
-\varepsilon u^{\prime \prime}-u u^{\prime}+u=p(x), \quad u(0)=0, \quad u(1) \approx 2,
$$

where $p(x)$ is given in such a way that the solution is

$$
u_{e}(x)=x+1-\exp (-x / \varepsilon)
$$

Although this problem is more general than the problem (2), we shall use it to compare the numerical solution obtained by the method from this Section with the exact one. Note that $u_{\varepsilon}$ behaves in the way described by Theorem 1 .

Let

and

$$
\begin{aligned}
E_{\infty} & =\max _{1 \leqslant i \leqslant n-1}\left|u_{e}\left(x_{i}\right)-w_{\varepsilon, i}\right| \\
E_{h} & =\left\|u_{\varepsilon, h}-w_{\epsilon, h}\right\|_{h} .
\end{aligned}
$$

In Table 1 we present the results of our method. The function $\lambda$ is taken with $\alpha=0.5$ and $\beta=1$. For $\varepsilon=10^{-2}$ this gives about $40 \%$ of the points $x_{i}$ in the interval $[0, \varepsilon]$ representing the layer. The percentage changes as $\varepsilon$ does: for $\varepsilon=10^{-3}$ it is about $33 \%$, for $\varepsilon=10^{-6}$ - about $26 \%$, and for $\varepsilon=10^{-9}$ - about $25 \%$. By changing $\alpha$ and $\beta$ one can change the density of the points $x_{i}$ in the layer and a prescribed percentage can be achieved, see $[9,10]$.

Table 1 confirms our theoretical results, but it shows the uniform pointwise convergence as well. This is the advantage of our method in comparison with the numerical solution obtained on equidistant meshes without transforming the original problem. 
These results are given in Table 2 and they also show the uniform convergence in the norm $\|\cdot\|_{h}=h\|\cdot\|_{1}$ (and even more than that). However, the uniform pointwise convergence is absent.

Table 1

\begin{tabular}{c|c|c|c|c|c|}
$n$ & $\varepsilon$ & $1 .-2$ & $1 .-3$ & $1 .-6$ & $1 .-9$ \\
\hline \multirow{2}{*}{50} & $E_{\infty}$ & $6.41-2$ & $7.44-2$ & $7.86-2$ & $7.88-2$ \\
& $E_{h}$ & $3.30-2$ & $3.48-2$ & $3.53-2$ & $3.54-2$ \\
\hline \multirow{2}{*}{100} & $E_{\infty}$ & $3,30-2$ & $3.82-2$ & $4.03-2$ & $4.03-2$ \\
& $E_{h}$ & $1.69-2$ & $1.78-2$ & $1.81-2$ & $1.81-2$ \\
\hline \multirow{2}{*}{200} & $E_{\infty}$ & $1.68-2$ & $1.94-2$ & $2.04-2$ & $2.04-2$ \\
& $E_{h}$ & $8.55-3$ & $9.02-3$ & $9.17-3$ & $9.19-3$ \\
\hline
\end{tabular}

Table 2

\begin{tabular}{c|c|c|c|}
$n$ & $\varepsilon$ & $1 .-2$ & $1 .-3$ \\
\hline 50 & $E_{\infty}$ & 0.200 & $4.02-2$ \\
& $E_{h}$ & $8.78-3$ & $3.60-3$ \\
\hline 100 & $E_{\infty}$ & 0.106 & $8.97-2$ \\
& $E_{h}$ & $4.66-3$ & $2.38-3$ \\
\hline 200 & $E_{\infty}$ & $5.22-2$ & 0.170 \\
& $E_{h}$ & $2.20-3$ & $1.74-3$ \\
\hline
\end{tabular}

REFERENCES

[1] L. Abrahamsson and S. Osher, 'Monotone difference schemes for singular perturbation problems', SIAM J. Numer. A nal. 19 (1982), 979-992.

[2] K.W. Chang and F.A. Howes, Nonlinear Singular Perturbation Phenomena: Theory and Application (Springer Verlag, New York, Berlin, Heidelberg, Tokyo, 1984).

[3] V.D. Liseikin and N.N. Yanenko, 'On the numerical solution of equations with interior and exterior boundary layers on a nonuniform mesh', in Proc. BAIL III Conference, Editor J.J.H. Miller (Bool Press, Dublin, 1984). pp. 68-80.

[4] J. Lorenz, 'Nonlinear boundary value problems with turning points and properties of difference schemes', in Lect. Notes in Math. 942, Editors W. Eckhaus and E.M. de Jager (Springer Verlag, Berlin, Heidelberg, New York, 1982). pp. 150-169. 
[5] J. Lorenz, 'Numerical solution of a singular perturbation problem with turning points', in Lect. Notes in Math. 1017, Editors H.W. Knobloch and K. Schmitt (Springer Verlag, Berlin, New York, 1983). pp. 432-439.

[6] J. Lorenz, 'Analysis of difference schemes for a stationary shock problem', SIAM J. Numer. Anal. 21 (1984), 1038-1053.

[7] K. Niijima, 'On a difference scheme of exponential type for a nonlinear singular perturbation problem', Numer. Math. 46 (1985), 521-539.

[8] R. Vulanović, 'On a numerical solution of a type of singularly perturbed boundary value problem by using a special discretization mesh', Univ. u Novom Sadu Zb. Rad. Prirod. -Mat. Fak. Ser. Mat. 13 (1983), 187-201.

[9] R. Vulanović, D. Herceg and N. Petrović, 'On the extrapolation for a singularly perturbed boundary value problem', Computing 36 (1986), 69-79.

[10] R. Vulanovic, 'A uniform numerical method for quasilinear singular perturbation problems without turning points', Computing (to appear).

Institute of Mathematics

University of Novi Sad

ul. Dr I Djuricica 4

Yu-21000 Novi Sad

Yugoslavia 